\title{
Thyroid function test - interpretation peril
}

\begin{abstract}
Thyroid dysfunction is one of the most common endocrine disorder leading to obesity .Due to its diverse clinical presentation physicians has to rely on thyroid function test. Thus physicians must be aware of the conditions affect the normal value of thyroid hormone.
\end{abstract}

Keywords: thyroid function test, pre analytical variables
Volume 7 Issue I - 2019

\author{
Rateesh Sareen \\ Department of Pathology \& Transfusion Medicine Santokba \\ Durlabhji Memorial Hospital Cum Research Center, India \\ Correspondence: Rateesh Sareen, Assistant Consultant, \\ Department of Pathology \& Transfusion Medicine \\ Santokba Durlabhji Memorial Hospital cum Research Center, \\ Jaipur, India, Tel 94I421647I, \\ Email drrateeshsareen@yahoo.co.in
}

Received: November 15, 2018 | Published: January 03, 2019

\section{Mini review}

Thyroid gland is a butterfly shaped gland situated below the larynx on either side and anterior to trachea at the level of second and third tracheal rings. ${ }^{1}$ The main hormones secreted by thyroid gland are Thyroxine (T4) and Triiodothyronine (T3). T4 constituting the majority of portion. Hormones secreted by thyroid are essential for normal development, differentiation, and metabolic balance. The main constituent of thyroid hormone is Iodine which enters in the gland either as inorganic or in organic form. Iodine along with peroxidase enzymes at colloid cell interface forms iodothyroninesmonoidothyronine, diidothyronine, triiodothyronine (T3) and thyroxin (T4). Hormone T4 is only secreted by thyroid gland whereas T3 is also secreted by peripheral tissue by deiodinase enzyme. The deiodinase enzyme system produces the inactive form ofT 3 that is RT3. The thyroid hormones circulate as protein bound form thyroid binding globulin TBG, transthyretin (TTR), TBPA, Albumin and free form. ${ }^{2,3}$ Thyroid prolife test is one of the commonest test ordered by physicians.

In the era of evidence based medicine laboratory should estimate analyte with accuracy and precision as they form the basis of clinical diagnosis and patient treatment. Therefore, it is essential that pathologists and clinicians are aware of various pre analytical factors affecting thyroid testing so that proper interpretation of tests can be done and consequent clinical decisions in the interest of patient can be done.

The laboratory testing of any analyte comprises of three phases namely- Preanalytical, Analytical and Post analytical phases. The preanalytical errors are the errors that occur from the time a laboratory test is ordered by the clinician until the sample is ready for analysis.
The preanalytical consists of various modifiable and non modifiable factors including age, pregnancy, medications, systemic and genetic diseases. In children the hypothalamic pitutary thyroid axis matures throughout infancy and childhood until puberty is reached. ${ }^{4}$ Thus both TSH and FT4 concentration are higher in children of first week of life up to one year of age. This fact is important to recognize as it can lead to under diagnosis of any case of congenital hypothyroidms.TSH levels tends to increase with every decade of life. Thus age related normal reference limits should be used for all thyroid tests. ${ }^{5}$

The clearance by the liver during the first trimester of pregnancy of the sialylated thyroxine-binding globulin, which is induced by estrogens, is decreased. Hence, the level of thyroxine-binding globulin increases, leading to the well known increases in serum thyroxine and triiodothyronine levels during pregnancy. Thus higher cut offs for T3 and T4 and lower cut offs for TSH are suggested during pregnancy.

In pregnancy there is an increase in the glomerular filtration rate, increasing in the clearance of iodide and a transfer of iodide and iodothyronines to the fetus. As a result, the inorganic iodide level in serum decreases to such an extent that pregnant women with marginal iodine intakes of less than $50 \mu \mathrm{g} / \mathrm{d}$ develop iodine deficiency and, in turn, enlargement of the thyroid gland. ${ }^{6}$

Physicians need to be aware of the effect of medications on thyroid. These drugs do not interfere chemically with test but affects metabolism of hormone product and release except for heparin. Heparin causes an increase in blood free thyroid hormone (FT4) levels. This is an important phenomenon to recognize as it can lead to spurious FT4 results. Effects of drugs on thyroid function test are shown in Table 1.

Table I Effect of drugs on thyroid testing

\begin{tabular}{ll}
\hline Drug & Effect on thyroid parameter \\
\hline Lithium, sulfonylureas & $\downarrow$ FT4, $\downarrow$ FT3, $\uparrow$ TSH \\
Glucorticoids, propranolol, amiodarone, propylthiouracil & $\downarrow$ FT3 leads to $\uparrow$ FT4 \\
Iodine lithium, dopamine antagonists, cimetidine & $\uparrow$ TSH \\
Glucocorticoids, dopamine agonists, somatostatin & $\downarrow$ TSH \\
Phenytoin, sulfonylureas, diazepam, furosemide, salicylates & $\uparrow \mathrm{FT} 4, \uparrow$ FT3 \\
Cholestyramine, ferrous sulfate, aluminum hydroxide & $\downarrow$ FT4, $\uparrow$ TSH \\
\hline
\end{tabular}


The advent of modern instruments analytical variation have minimal interference with any compound either heterophile antibodies or endogenous thyroid antibody. Heterophile antibodies are those types of antibodies in serum that have been induced by infections or exposure to therapeutic agents containing specific antigens or by unintentional immunization through exposure to animals in the workplace (e.g. pet handlers, sheep herdsmen, etc.). They may also be poly reactive antibodies such as IgM rheumatoid factor. These heterophile antibodies may cross-react with any of the thyroid function test assay methods (immunoassay-based, utilizing animal antigens) leading to false results, which are more often inappropriately high. It is advisable to request a retest by another laboratory that performs test by a different immunoassay method containing a different animal antibody source (e.g. from mouse to sheep in the case of TSH; from rabbit to goat in the case of FT4).

To estimate TFTs, serum is preferred specimen and ideally whole blood samples should be allowed to clot for more than $30 \mathrm{~min}$ and then centrifuged and separated. Serum can be stored at $4-8^{\circ} \mathrm{C}$ for up to 7 days. Storage at $-20^{\circ} \mathrm{C}$ is recommended if the assay is to be delayed for more than 1 week. Collection of serum in barrier gel tubes does not affect the results of most TFTs. Generally, thyroid hormones are quite stable whether stored at room temperature, in refrigerator or frozen. Furthermore, TSH and T4 in dried whole blood spots used to screen for neonatal hypothyroidism are also stable for months when stored with a desiccant. Similarly, hemolysis, hyperlipidemia, and hyperbilirubinemia do not produce interference in hormone estimation by different assays.?

\section{Post analytical variations}

Post analytic errors are comparatively less frequent when compared with the preanalytical and analytical variations. This is subdivided into the intra-laboratory (procedures done after conducting the test) and departmental (analysis of the result by the user). ${ }^{8}$

\section{Intra-laboratory phase:}

a. Wrongful data entry - reporting a correct value in different patient.

b. Wrong entry of the units - $\mathrm{mg} / \mathrm{L}$ instead of $\mathrm{mg} / \mathrm{dL}$.

c. Data entry errors-20 instead of 2.0 .

d. Normal range not derived from the local population.

\section{Post laboratory phase:}

a. Delayed access of the test results due to excessive turnaround time.

b. Underutilization of the test results for lack of understanding.

c. Failure to interpret correctly (high T4 is normal in pregnancy).

d. Failure to understand the physiology (delay in TSH rise after anti thyroid drugs).

\section{Acknowledgments}

None.

\section{Conflict of interest}

The author declares there is no conflict of interest.

\section{References}

1. Ganong FW. Review of Medical Physiology. California: Appleton and Lange; 1995. pp. 314.

2. Garg MK, Namita Mahalle, Hari Kumar KVS. Laboratory evaluation of thyroid function: Dilemmas and pitfalls. Medical Journal. 2016;9(4):430-436.

3. Li M, Eastman CJ. The changing epidemiology of iodine deficiency. Nat Rev Endocrinol. 2012;8(7):434-440.

4. Fisher DA, Nelson JC, Carlton EI, et al. Maturation of human hypothalamic-pituitary-thyroid function and control. Thyroid. 2000;10(3):229-234.

5. Surks MI, Boucai L. Age- and race-based serum thyrotropin reference limits. J Clin Endocrinol Metab. 2010;95(2):496-502.

6. Narayanan S. Current concepts of thyroid function and laboratory evaluation. Indian J Clin Biochem. 1997;12:25-34.

7. Demers LM, Spencer CA. Laboratory medicine practice guidelines: Laboratory support for the diagnosis and monitoring of thyroid disease. Clin Endocrinol (Oxf). 2003;58(2):138-140.

8. Plebani M. Errors in clinical laboratories or errors in laboratory medicine? Clin Chem Lab Med. 2006;44:750-759. 